\title{
TB sequel: incidence, pathogenesis and risk factors of long-term medical and social sequelae of pulmonary TB - a study protocol
}

Andrea Rachow ${ }^{1,2+}$, Olena Ivanova ${ }^{1,2^{*}+}$ (D) Robert Wallis ${ }^{3}$, Salome Charalambous ${ }^{3}$, llesh Jani ${ }^{4}$, Nilesh Bhatt ${ }^{4}$, Beate Kampmann ${ }^{5,6}$, Jayne Sutherland ${ }^{5}$, Nyanda E. Ntinginya ${ }^{7}$, Denise Evans ${ }^{8}$, Knut Lönnroth ${ }^{9}$, Stefan Niemann ${ }^{10}$, Ulrich E. Schaible ${ }^{10}$, Christof Geldmacher ${ }^{1,2}$, lan Sanne ${ }^{8,11}$, Michael Hoelscher ${ }^{1,2}$ and Gavin Churchyard 3,12,13

\begin{abstract}
Background: Up to fifty percent of microbiologically cured tuberculosis (TB) patients may be left with permanent, moderate or severe pulmonary function impairment. Very few studies have systematically examined pulmonary outcomes in patients to understand the pathophysiologic basis and long-term socio-economic consequences of this injury. The planned multi-country, multi-centre observational TB cohort study, aims to advance the understanding of the clinical, microbiological, immunological and socio-economic risk factors affecting long-term outcome of pulmonary TB. It will also determine the occurrence of reversible and irreversible socio-economic consequences to patients, their households and the health sector related to pulmonary TB disease and its treatment.
\end{abstract}

Methods: We will enrol up to 1.600 patients with drug sensitive and multidrug-resistant pulmonary TB who are treated according to the local standard of care by the respective National TB Program. Recruitment is taking place at the time of TB diagnosis at four African study clinics located in The Gambia, Mozambique, South Africa and Tanzania. The primary outcome is the proportion of TB patients with severe lung impairment measured by spirometry at 24 months after TB treatment initiation. Biological samples, including sputum, urine and blood, for studying host- and pathogenic risk factors will be collected longitudinally and examined in a nested case-control fashion. A standardized quality of life questionnaire will be used together with a novel version of WHO's generic patient cost instrument which has been adapted for the longitudinal study design.

Discussion: This study is an integral part of an overall strategy to fill a knowledge gap needed to improve TB treatment outcomes globally. The main scientific goal is to identify the major pathogenic mechanisms associated with poor TB treatment outcomes, so that such pathways can be interrupted to avert long term TB sequelae. National as well as supra-national stakeholders and decision makers have been integrated early in the study planning process to inform future treatment guidelines and national health policies.

Trial registration: ClinicalTrials.gov: NCT03251196, August 16, 2017.

Keywords: Tuberculosis, Tuberculosis outcome, Sequelae, Lung function, Lung impairment, Patient costs, Study protocol, Cohort, Africa, Risk factors, Treatment outcome

\footnotetext{
* Correspondence: olena.ivanova@|rz.uni-muenchen.de

${ }^{\dagger}$ Andrea Rachow and Olena Ivanova contributed equally to this work.

'Division of Infectious Diseases and Tropical Medicine, Medical Centre of the

University of Munich (LMU), Munich, Germany

${ }^{2}$ German Center for Infection Research (DZIF), Partner Site Munich, Munich,

Germany

Full list of author information is available at the end of the article
}

(c) The Author(s). 2019 Open Access This article is distributed under the terms of the Creative Commons Attribution 4.0 International License (http://creativecommons.org/licenses/by/4.0/), which permits unrestricted use, distribution, and reproduction in any medium, provided you give appropriate credit to the original author(s) and the source, provide a link to the Creative Commons license, and indicate if changes were made. The Creative Commons Public Domain Dedication waiver (http://creativecommons.org/publicdomain/zero/1.0/) applies to the data made available in this article, unless otherwise stated. 


\section{Background}

Tuberculosis (TB) remains one of the world's deadliest communicable diseases. In 2016, an estimated 10.4 million people developed $\mathrm{TB}$ and 1.3 million died from the disease [1]. TB related morbidity and mortality remain particularly high in African countries, mainly due to the impact of HIV, sustained poverty and food insecurity as well as due to treatment challenges including the rise in drug-resistant TB [2].

For the past 40 years, treatment success in tuberculosis has been defined as the eradication of active infection whilst preventing resistance and recurrence, achieved through multidrug antimicrobial treatment. Current estimates of the global TB disease burden include incidence and prevalence of active TB, TB death rates, and disability-adjusted years of life (DALY) lost due to active TB, but do not consider DALYs lost due to long-term disability due to TB sequelae or reduced longevity in patients considered cured [3, 4]. However, accumulating evidence indicates that permanent lung injury due to TB is frequent and substantial. In one study in Papua New Guinea, the mean FEV1 (the maximal volume of air exhaled in the first second after a full inspiration), was reduced to $64 \%$ of that of healthy controls at the time of TB diagnosis [5]. FEV1 improved by $11 \%$ after 2 months of TB treatment, but did not further improve subsequently. At the conclusion of successful anti-microbial treatment, $27 \%$ of TB patients still had moderate or severe impairment of pulmonary function. [5]. Another study from India found severe and irreversible obstructive pulmonary ventilation defects in one-third of TB patients one year after completion of TB treatment [6]. A literature review of South African studies showed that lung function impairment and chest symptoms were consistently associated with pulmonary TB [7]. In the United States, studies demonstrated that even persons cured from TB have considerably shortened life expectancy $[5,6]$. There is limited evidence on pulmonary function impairment in African populations outside South Africa and presently no estimates of the impact of TB on life expectancy in African patients. Further, associated risk factors contributing to permanent lung function impairment after TB are unknown.

\section{Clinical, environmental and behavioural risk factors}

The risk of developing TB is up to 30 times greater in people living with HIV than among those without HIV infection [8]. TB patients with advanced AIDS are at increased risk of immune reconstitution inflammatory syndrome (IRIS) and death. The long-term effects of HIV and antiretroviral treatment on lung function, however, are not known. Diabetes mellitus is also recognized as a $\mathrm{TB}$ risk factor and the African region is experiencing an increasing prevalence of diabetes alongside other non-communicable diseases [9-11]. However, little is known about the potential impact of diabetes on long-term TB outcomes. Other risk factors that can potentially have long term effects include pre-existing pulmonary conditions like Chronic Obstructive Pulmonary Disease (COPD) and asthma, and associated risk factors such as smoking, indoor air pollution and harmful alcohol use $[12,13]$. Malnutrition is also an important risk factor for TB [14], however, the association with sequelae after treatment has not been studied.

\section{Host-immune response and associated risk factors}

It is well known that host responses to Mycobacterium tuberculosis (MTB) also contribute to lung pathology through excessive induction of the inflammatory pathway. However, whether the systemic host response before and after TB treatment initiation can be used to predict pathology and treatment outcomes is largely unknown. Blood and sputum-derived neutrophil activation and inflammatory mediators will be examined in order to elucidate the predominant pathway of lung inflammation in TB and inform on future host-adjunctive therapy strategies. For example, polymorpho-nuclear neutrophilic granulocytes have been associated with active tuberculosis and the failure to control the infection, and likely contribute to exacerbated pathology and long term sequelae and lung function loss [15-17]. Additionally, there are very few studies that have assessed specific host biomarkers in clinical research studies, such as the T-cell activation marker-tuberculosis (TAM TB) assay [18], which have shown a correlation with diagnosis and treatment response in TB [19].

\section{Pathogen diversity and microbial risk factors}

There is increasing evidence that genomic diversity of the MTB complex influences important patho-biological properties, such as transmissibility and pathogenicity, and affects host-immune responses and clinical manifestations [20, 21]. Moreover, microbial factors are recognized as important determinants of microbiologic outcomes such as treatment failure or relapse. Baseline drug resistance can affect the risk of new (acquired) resistance and subsequent treatment failure. For some drugs, such as rifampin, this effect can be profound [22]. Sputum bacillary burden at baseline and culture status after 2 months of treatment affect relapse risk [23]. However, very little is known about the contributions of all these and other microbial factors, such as strain genetic background or the presence of mixed infections, on long-term pulmonary function outcomes.

Socio-economic determinants and costs of tuberculosis Several previous studies have documented high patient costs before patients are diagnosed and during TB 
treatment $[24,25]$, where patients may lose about half of their household income due to TB. Loss of income and direct expenses may result in poorer treatment outcomes, including increased risk of TB infection and progression, the development of drug resistance and long term pulmonary impairment. The WHO End TB Strategy has included an microeconomic target, that no TB patient or affected household should experience catastrophic expenditure or costs related to TB illness and TB care [26], and a generic protocol for the measurement of catastrophic costs due to TB has been developed [27]. Measuring catastrophic cost is a useful indicator of equality in advancing towards universal health coverage. Although patient costs during diagnosis and TB treatment has been documented there is little evidence on the costs and socioeconomic consequences of TB after treatment has been completed and how they are associated to different clinical TB outcomes. In addition, the published literature contains surprisingly little evidence about the costs to the health systems of treating TB and TB sequelae, especially in Africa.

\section{Study aim}

TB Sequel project aims to: i) advance understanding of the clinical, microbiological, immunological and socioeconomic risk factors affecting or predicting the longterm pulmonary function outcome; ii) determine costs on patient and health system level related to TB disease, $\mathrm{TB}$ treatment and TB sequelae.

\section{Methods/design}

\section{Study partners and setting}

The TB Sequel cohort study is conducted by the TB Sequel consortium including a number of research institutions based in Africa and Europe (Additional file 1). The Division of Infectious Diseases and Tropical Medicine at the University of Munich (LMU), Germany and The AURUM Institute in Johannesburg, South Africa coordinate the project. The TB Sequel cohort study will recruit TB patients in four African countries of which three belong to high TB burden countries. In Johannesburg, South Africa, the study is conducted by the University of Witwatersrand (WITS) at the Clinical HIV Research Unit (CHRU) located at the Helen Joseph Hospital that serves an urban area with approximately one million inhabitants. In Mbeya, Tanzania, the study takes place at the National Institute of Medical Research - Mbeya Medical Research Centre (NIMR-MMRC). The NIMR-MMRC is located within the same premises of the Mbeya Zonal Referral Hospital (MZRH), one of the four largest referral hospitals in Tanzania, which serves a total population of about 500.000 inhabitants. In Maputo, Mozambique, the Instituto Nacional de Saúde (INS) is conducting the TB Sequel cohort study at two research units, first located in
Mavalane Health Centre (City of Maputo) serving approximately 620.000 inhabitants, and second, located in Machava General Hospital (Province of Maputo) where the majority of MDR-TB patients of Maputo are treated. In The Gambia, this study is conducted by the Medical Research Council (MRC) Unit The Gambia in the Greater Banjul area, where two thirds of all TB patients diagnosed in the country present. The Research Center Borstel (FZB) in Germany is responsible for MTB isolates typing and contributes to host responses studies including training of African researchers. The socio-economic sub-study is being led by the Health Economics and Epidemiology Research Office ( $\left.\mathrm{HE}^{2} \mathrm{RO}\right)$ at WITS University in South Africa in collaboration with the Department of Public Health Sciences at Karolinska Institutet, Sweden and WHO Geneva.

\section{Study design}

We will conduct a prospective, multi-country, multicentre, observational cohort study and recruit up to 1.600 pulmonary $\mathrm{TB}$ patients at the time of $\mathrm{TB}$ diagnosis who will be followed during and after $\mathrm{TB}$ treatment. At enrolment, TB diagnosis is based on sputum tested positive for the presence of MTB either by Xpert MTB/RIF (or Ultra) or culture methods (Fig. 1). At baseline and at defined study visits (see Schedule of Events (SOE) Table 1) clinical data, data on risk factors, information on comorbidities, socio-economic data, as well as biological samples (sputum, urine and blood) will be collected from all participants. This large cohort will therefore serve as a platform for all nested research activities related to immunological, microbiological and socio-economic studies as well as future therapeutic interventions (Additional file 2).

\section{Participant eligibility}

For enrolment, each study participant must meet all of the inclusion criteria and none of the exclusion criteria summarized in Table 2.

\section{Duration of study}

Recruitment commenced in September 2017 and is expected to continue until September 2019. Follow-up will last for a minimum of 24 months in all participants to ensure that any loss in reported lung function will have become chronic during follow-up [28]. Further, the chronicity and final degree of long term costs and comorbidities resulting from TB disease or treatment shall be confirmed by a follow up period of at least 24 months length. End of study is expected in September 2021.

\section{Primary outcome and study endpoints}

The primary outcome is a proportion of TB patients with severe pulmonary function impairment (restrictive, obstructive or mixed ventilation impairment), measured 


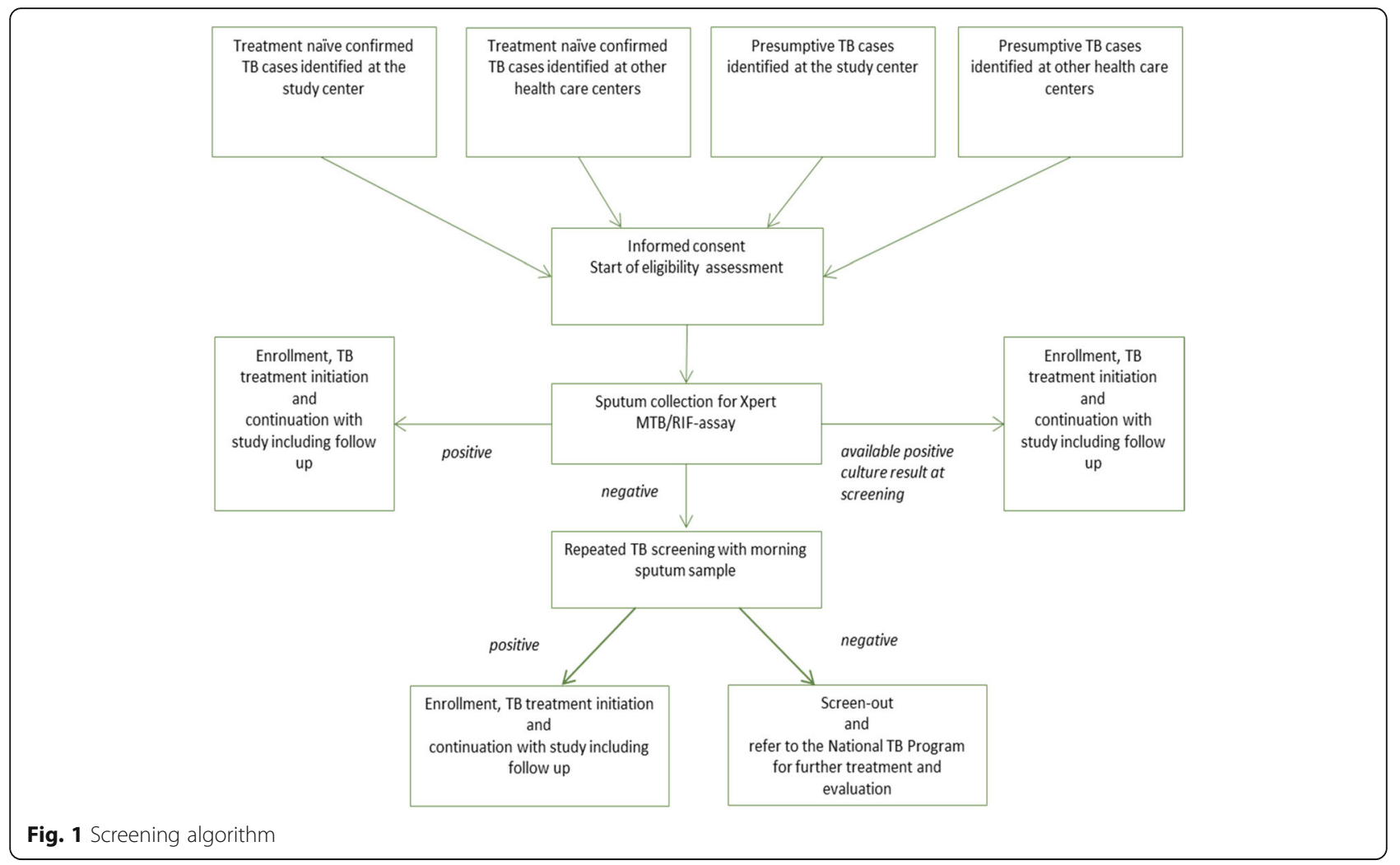

by spirometry at 24 months from TB treatment initiation and compared to national reference values. Long term lung function impairment is considered a clinically meaningful condition contributing to long term morbidity and mortality in former TB patients.

Amongst secondary outcomes are: the proportion of subjects with any impairment of pulmonary function as well as severity and type of impairment at TB diagnosis and 2, 6, 12 and 18 months; proportion of subjects with liquid and solid MTB culture conversion at different time points during or at the end of anti-TB treatment; the frequency of all-cause hospitalization episodes and all-cause mortality during follow-up of 24 months; the proportion of subjects with recurrent TB and; the proportion of subjects suffering from ongoing non-pulmonary comorbidities which are related to $\mathrm{TB}$ disease and treatment at different time points during 24 months of follow-up.

Studies regarding host immunology- and pathogen-associated risk factors and markers are planned as explorative, nested, case-control studies. Sample selection will be based on specific outcomes and risk factors present in the study participants.

Main outcomes studied in the context of socio-economic research are the proportion of subjects incurring catastrophic cost due to TB by the end of treatment; changes in income within follow up period; and scales for quality of life and general disability at treatment start, end of treatment and at follow-up. The cost of providing TB care and treatment, will be estimated from the health provider perspective, for both drug sensitive and drug resistant TB.

\section{Sample size}

We hypothesized that the prevalence of the primary endpoint - severe lung impairment at 24 months period of follow up after TB diagnosis (conservative estimation based on a literature review) will be $15 \%$. A sample size of 1.600 patients allows the estimation of the primary end point in the total cohort with a precision (half width of the $95 \%$ confidence interval) of about $1.75 \%$. In addition, the prevalence within specific subgroups can be estimated adequately, e.g. with a precision between 2.5 and $7.8 \%$ in subgroups with a proportion of 50\% (e.g. HIV) and 5\% (e.g. diabetes) of the total cohort, respectively. Moreover, based on a pre-set alpha of $5 \%$ (two-sided) the sample size allows the detection of risk factors causing a duplication of the prevalence of severe lung impairment with a power of at least $80 \%$, even for risk factors with a low prevalence of $5 \%$ in the total cohort. Depending on the availability and quality of patient's end-point data, or if the prevalence of the primary outcome is less than $15 \%$, it may be necessary to recruit more or less participants into the study.

\section{Recruitment and screening}

Recruitment takes place at the research sites and health care facilities through collaboration with National TB 
Table 1 Schedule of Events (SOE)

\begin{tabular}{|c|c|c|c|c|c|c|c|c|c|c|}
\hline $\begin{array}{l}\text { Activities } \\
\text { Visits }\end{array}$ & Screening & Baseline & Day 14 & Month 2 & Month 4 & Month 6 & Month 9 & Month 12 & Month 18 & Month 24 \\
\hline Eligibility assessment & $x$ & & & & & & & & & \\
\hline Xpert MTB/RIF (Ultra) assay & $x$ & & & & & & & & & \\
\hline Sputum smear and culture & $x$ & $x$ & & $x$ & $x$ & $x$ & $(x)$ & $(x)$ & $(\mathrm{x})$ & $(x)$ \\
\hline Drug sensitivity testing & & $x$ & & & & & & & & \\
\hline Demographic data & & $x$ & & & & & & & & \\
\hline Clinical examination & & $x$ & $x$ & $x$ & $x$ & $x$ & $x$ & $x$ & $x$ & $x$ \\
\hline Chest X-ray & & $x$ & & & & $x$ & & & & $x$ \\
\hline Lung function assessment & & $x$ & & $x$ & & $x$ & $x$ & $x$ & $x$ & $x$ \\
\hline ECG & & $x$ & & & & & & $x$ & & $x$ \\
\hline Medical history (update) & & $x$ & $x$ & $x$ & $x$ & $x$ & $x$ & $x$ & $x$ & $x$ \\
\hline TB treatment and adherence & & & $x$ & $x$ & $x$ & $x$ & $(x)$ & $(x)$ & $(x)$ & $(x)$ \\
\hline $\begin{array}{l}\text { Quality of life/patient's cost } \\
\text { questionnaire }\end{array}$ & & $x$ & & $x$ & & $x$ & & $x$ & & $x$ \\
\hline HIV test (CD4 count) & & $x$ & & & & & & $x$ & & $x$ \\
\hline Hepatitis B serology & & $x$ & & & & & & & & \\
\hline Haematology and Biochemistry & & $x$ & & & & $x$ & & $x$ & & $x$ \\
\hline Urine dip stick test & & $x$ & & $x$ & $x$ & $x$ & & & & \\
\hline $\begin{array}{l}\text { Blood storage for nested } \\
\text { research studies }\end{array}$ & & $x$ & $x$ & $x$ & $x$ & $x$ & $x$ & $x$ & & \\
\hline $\begin{array}{l}\text { Sputum storage for nested } \\
\text { research studies }\end{array}$ & & $x$ & $x$ & $x$ & $x$ & $x$ & $(\mathrm{x})$ & $(x)$ & $(x)$ & $(x)$ \\
\hline $\begin{array}{l}\text { Urine storage for nested } \\
\text { research studies }\end{array}$ & & $x$ & & $x$ & $x$ & $x$ & $(\mathrm{x})$ & $(x)$ & $(x)$ & $(\mathrm{x})$ \\
\hline
\end{tabular}

Program (NTP), and in the communities which might be enhanced by individual and community awareness through advertisement, posters, radio announcements, and community sensitization campaigns. Each study site implements its own strategy to enhance recruitment.

\section{Study procedures}

Clinical and pulmonary assessments, biological sample collections and collection of socio-economic data will be performed according to the pre-defined SOE. All enrolled patients will be treated within the NTP and

Table 2 Inclusion and exclusion criteria

\begin{tabular}{|c|c|c|}
\hline & Inclusion criteria & Exclusion criteria \\
\hline 1 & $\begin{array}{l}\text { At least one sputum sample tested positive for MTB by Xpert MTB/RIF } \\
\text { assay in the study clinic/study laboratory or at least one sputum } \\
\text { sample tested positive by culture methods in study laboratory or } \\
\text { other TB laboratory }\end{array}$ & Anti-TB treatment in the last 6 months $^{a}$ \\
\hline 2 & Be $\geq 18$ years of age & $\begin{array}{l}\text { Incapacity to produce and provide two sputum samples } \\
\text { of sufficient volume and quality }\end{array}$ \\
\hline 3 & $\begin{array}{l}\text { Willing to provide a written consent or witnessed oral consent in the } \\
\text { case of illiteracy for participation in the study, prior to patient's first } \\
\text { sample or other study-specific data being collected }\end{array}$ & $\begin{array}{l}\text { Severe medical or psychiatric condition which in the opinion of the } \\
\text { site investigator or designee, might interfere with the ability to give } \\
\text { true informed consent and to adhere to the study requirements }\end{array}$ \\
\hline 4 & Willing to be tested for HIV infection & Currently imprisoned \\
\hline 5 & $\begin{array}{l}\text { Agreeing to the collection and storage of blood, urine, and } \\
\text { sputum specimens }\end{array}$ & $\begin{array}{l}\text { Taking part in investigational product trials related to TB and/or } \\
\text { lung diseases }\end{array}$ \\
\hline 6 & Willing to start anti-TB treatment after TB diagnosis & \\
\hline 7 & $\begin{array}{l}\text { Living within the study area and willing to inform the study team } \\
\text { of any change of address during the treatment and follow up period }\end{array}$ & \\
\hline
\end{tabular}

${ }^{a}$ To ensure that no treatment failure are recruited into (immunological or genetic) marker studies

${ }^{\mathrm{b}}$ To ensure sufficient amount of sputum for host- and mycobacterial marker studies 
receive anti-TB treatment according to the local national guidelines.

\section{Clinical assessments and procedures}

We will assess the pulmonary outcome, co-morbidities and clinical risk factors using a number of methods. These include: spirometry using the EasyOne or EasyOnPC (ndd Medical Technologies, Inc., Zurich, Switzerland), Electrocardiogram (ECG), 6 min walking test (6MWT), chest $\mathrm{X}$-ray, St. George's respiratory questionnaire, blood analysis (haematology, biochemistry including HbA1c, HIV/CD4, hepatitis B), urinalysis and risk-factors questionnaires (risk behaviour, environmental and occupational risk factors, among others). In addition, household air pollution is estimated by measuring participant's breath carbon monoxide (CO) output (exhaled CO levels), which is collected using a handheld breath $\mathrm{CO}$ monitor - The Microt ${ }^{\mathrm{Tm}}$ Smokerlyzer (Bedfont ${ }^{\circledR}$ Scientific Ltd., UK).

\section{Laboratory procedures and testing}

All laboratory procedures (apart from some specific assays planned for investigation of host-immune response or pathogen characteristics not locally available) will be performed at each study site and at the designated site laboratories under the supervision of the study principal investigators and using unified standard operating procedures. In the study TB labs we will carry out standard TB diagnostic procedures including smear, Xpert MTB/ RIF or Ultra assay (Cepheid Inc., USA), liquid and solid culture, drug resistance testing in culture and molecular speciation testing. For analysis of pathogen-related risk factors, we will perform a number of pilot studies including characterization of MTB strains by next generation whole genome sequencing, identification of mixed infections and minority subpopulations as well as capture the bacterial load, e.g. using molecular methods to monitor treatment response. A number of different assays will be performed to study in detail the host response to TB treatment and individual predisposing risk factors for long term lung outcome on stored samples, such as (but not limited to) sputum and whole blood transcriptomics, flow cytometry, cytokine assays and host-genetic analysis.

\section{Assessments related to socio-economic consequences and quality of life}

All patients will be interviewed by trained study staff using a novel version of the WHO's generic TB patient cost survey instrument [27] which has been adapted for both the longitudinal study design and local context of each setting. The instrument includes questions about direct out-of-pocket costs (net of reimbursement) of medical care, transport, food and accommodation during health seeking, indirect costs such as income loss and socio-economic coping mechanisms (e.g. taking a loan, selling assets or property or taking children out of school). Moreover, the instrument collects information about health insurance coverage and reimbursements and social welfare or paid sick leave received. A standardized short-form 36 item health survey (SF-36) combined with a PIQ- ${ }^{\text {Tix }}$ (4 items) will simultaneously measure overall health, pain severity and the impact on functional health and well-being. In addition to the above we will also be using the Sheehan Disability scale (5 items) to assess health status impairment associated with TB and Kessler Psychological Distress scale (K10), a 10 item questionnaire, to measure distress based on questions about anxiety and depressive symptoms. These are all standardized validated questionnaires that have been widely used in TB studies and are simple to administer and repeatable over time. The patient cost and quality of life questionnaires will be completed at baseline and at pre-defined visits (e.g. 2, 6, 12 and 24 months). Health system costs will be based on actual patient usage with unit costs assigned, but will be collected later for the 24 month period. Resource utilization and treatment outcomes will be collected using clinical records, health facilities records, price lists and reports.

\section{Sample collection and storage}

Blood, sputum and urine specimens are obtained from each patient at different time points for analysis and storage. The SOE is aligned with The RePORT International cohort and other TB cohorts to enable future research collaborations with other TB research consortia and facilitate comparability of outcome data across different cohorts. The researchers who manage or have access to human specimen data are legally and ethically obliged to protect these data according to international standards. Specific samples, e.g. TB strains, will be shipped to collaborating institutions and stored in their laboratories for further analysis.

Stored specimens will also be accessible for evaluation of future emerging TB diagnostics, genetic markers and biomarkers, provided informed consent and ethical approval for future use are available. For any additional investigations not explicitly mentioned in the original study protocol, a request for study amendment will be submitted to the relevant ethics committees.

\section{Data management and statistical analysis}

A study specific database has been developed using a web-based Clinical Data Management System - Open Clinica ${ }^{\circ}$, for data entry and data cleaning, supervised by the lead data manager at LMU. All data collected at each performed visit must be entered in the respective electronic case report forms (eCRFs) provided in the data base.

The statistical analysis of this observational cohort study will be exploratory and use descriptive as well as 
inferential statistical methods. The statistical analyses will primarily be based on all enrolled patients with valid data available. Preliminary data sets will be analysed along the study in the frame of interim analyses, with the final analysis being completed once the database is locked.

\section{Study monitoring}

Study activities related to data generation, recording and management processes will be monitored on a regular basis. Two external monitors from coordinating institutions have been assigned to this task. These clinical monitors will follow the study closely, visit the sites at regular intervals and will be in contact by phone and written communication, as required. In addition to close supervision by the external monitors as well as by a local supervisor, one internal clinical monitor is supervising the procedures in the study clinics at each study site in the context of a continuing clinical monitoring training which includes workshops and distance learning modules. Additionally, an External Advisory Board consisting of six representatives of NTPs, local and international policy makers as well as relevant (clinical) scientists in the field of TB research was created to regularly evaluate the study activities and research progress as well as support the process in scientific decision making with regards to the global TB research agenda.

\section{Ethical considerations}

This study is performed in accordance with the study protocol, the Declaration of Helsinki (October 2013) and the WHO Handbook for Good Clinical Research Practice (July 2002) as well as any other applicable national and other regulatory guidelines. The protocol, Informed Consent Form (ICF), eCRFs and other relevant study documents were reviewed and approved by all respective Ethical Committees at each study site and also at coordinating institutions. All participants sign an ICF prior to enrolment in the study. For each enrolled subject, we will assign a unique study identification number, which will be used to identify the subject's data and sample within the project. The eCRFs and other study documents do not include any identifying information linked to the study ID in order to maintain confidentiality for all records and data of the participants.

\section{Discussion}

Current guidelines do not foresee specific monitoring or treatment of pulmonary injury related to $\mathrm{TB}$, nor has disability due to long term loss of lung function or other sequelae been included in current global estimates of the total burden of disease. Social support for TB patients rarely consider the period after cure has been declared. As NTP in most countries do not follow up patients after "cure", the extent of the long-term complications and their impact on individuals and households so far remain unknown.

The present lack of knowledge regarding the pathogenic mechanisms or clinical co-factors causing adverse outcomes in TB hinders the development and evaluation of interventions to reduce the overall TB disease burden or costs related to TB. Additionally, the lack of current, geographically relevant information about the economic impact of TB and the likely costs to national health systems pose an obstacle to efficient resource allocation both within TB programs and across competing health and social care priorities. Studies, such as TB Sequel, examining long term disability and associated risk factors, income loss, and treatment-related costs are crucial to inform the policy changes needed to address poor long-term TB outcome and catastrophic costs burden. Compared to other TB cohort studies TB Sequel will follow patients for at least 24 months which enables us to report lung outcome when any impairment related to TB disease has stabilized, thus when it can be assumed to have become chronic. Likewise the characteristics of ongoing socio-economic consequences and disease burden can be studied in those patients with relevant chronic lung or other TB related injury for at least 24 months. This allows for new estimates of TB disease burden which go beyond microbiological cure. Due to sample size and selection of different study sites across Africa we will be able to describe the influence of important potential risk factors on lung outcome such as infection with an MDR-TB strain, HIV-coinfection and comorbidities like COPD and diabetes but also the relevance of different genetic and socio-economic background. On the other hand, the follow-up of only 24 months precludes the investigation of interrelation of pulmonary TB with pre-existing and especially newly developed comorbidities as well as the influence of $\mathrm{TB}$ on long term survival and socio-economic status. Another limitation of the study is that the investigators have no influence on the composition of and adherence to anti-TB (and anti-retroviral) treatment and individual treatment regimens might have a different influence on lung outcome. On each study visit, co-medication and drug adherence are documented in order to enable sub-group analyses as well as to control for it during analysis of other risk factors for poor treatment outcomes. Finally, the capacities for adequate treatment and follow up for the majority of diagnosed comorbidities are currently limited or even not available at most study settings.

Thus, the TB Sequel Project is an integral part of an overall strategy to fill a knowledge gap needed to improve TB treatment and outcomes globally. It is an interdisciplinary project that combines multiple components: research, capacity building and translation of evidence 
into policies. All research sites will receive training and are included in capacity building activities (both in human resources and infrastructure) which creates an enabling environment for research and increases competencies in diagnosis and management of $\mathrm{TB}$ and lung health with direct benefit for future TB patients.

\section{Dissemination of results}

We anticipate that the results of this study will be relevant to a broader research community, health care providers and policy makers. Hence, we will disseminate the findings of this study through different channels, such as TB Sequel web page (www.tbsequel.org), scientific articles in international peer-reviewed journals, presentations at national and international conferences, social media and policy briefs. To further increase the impact of our findings, national and supra-national stakeholders and decision makers, including the World Health Organization, were consulted early in the study planning process and results will be regularly shared with these institutions to inform future treatment guidelines and national health policies. The dissemination of research results will be an integral part of the TB Sequel project which also focuses on collaboration strengthening through: developing new and intensifying existing links between involved research institutions by integrating and harmonizing common activities to create an enabling environment at African research institutions for the exchange of knowledge; participation of local, regional and international stakeholders in the project to facilitate translation of evidence from the TB Sequel study into policy and practice' and finally, the collaboration with other research networks such as PanACEA, The RePORT International, German Center for Infection Research (DZIF) projects and RESULTS Africa to ensure the relevance and impact of TB Sequel research activities.

\section{Additional files}

Additional file 1: TB Sequel Project partners and research sites.
(DOCX $276 \mathrm{~kb}$ )

Additional file 2: Overview diagram depicting the interrelation of the main TB-cohort and embedded sub-studies. (DOCX 91 kb)

\begin{abstract}
Abbreviations
AIDS: Acquired immune deficiency syndrome; CO: Carbon monoxide; COPD: Chronic obstructive pulmonary disease; DZIF: German Center for Infection Research; ECG: Electrocardiography; eCRF: Electronic clinical report form; FEV1: Forced expiratory volume in the first second; HIV: Human immunodeficiency virus; ICF: Informed consent form; IRIS: Immune reconstitution inflammatory syndrome; MTB: Mycobacterium tuberculosis; NTP: National TB Program; SOE: Schedule of events; TAM TB: T-cell activation marker-tuberculosis; TB: Tuberculosis; WHO: World Health Organization
\end{abstract}

\section{Acknowledgements}

We would like to recognize the hard work and valuable contributions of all colleagues and partners in this project. We want to specifically mention: Kavindhran Velen, Farzana Sathar, Maria Papathanasopoulos, Anna-Maria Mekota, Friedrich Rieß, Fidelina Zekoll, Ulrich von Both, Matthias Merker,
Christoph Leschczyk, Celso Khosa, Khalide Azam, Nhassengo Pedroso, Elton Uamusse, Nadia Sitoe, Mohammed Rassool, Issa Sabi, Elimina Siyame, Bariki Mtafya, Daniel Mapamba, Mkunde Chachage, Owolabi Olumuyiwa, Jayasooriya Shamanthi, Sillah Abdou, Sydney Rosen and Andrew Siroka. We are also thankful to representatives of NTPs and representatives of other national health authorities for their support from early stages of the project.

\section{Funding}

This project is funded by the German Ministry for Education and Research (BMBF) and is a part of the Research Networks for Health Innovations in Sub-Saharan Africa.

\section{Availability of data and materials}

Data sharing is not applicable as no datasets were generated or analysed for this protocol article.

\section{Authors' contributions}

AR, RW, SC, IJ, NB, BK, JS, NN, DE, KL, SN, US, CG, IS, MH, GC conceived and designed the study. AR and OI drafted the article. AR, OI, RW, SC, IJ, NB, BK, JS, NN, DE, KL, SN, US, CG, IS, MH, GC critically revised the article for important intellectual content. AR, OI, RW, SC, IJ, NB, BK, JS, NN, DE, KL, SN, US, CG, IS, MH, $\mathrm{GC}$ read and approved the final manuscript.

\section{Ethics approval and consent to participate}

The protocol, Informed Consent Form (ICF), eCRFs and other relevant study documents were reviewed and approved by all respective Ethical Committees at each study site:

WITS: Human Research Ethics Committee of the University of the Witwatersrand, Johannesburg.

INS: Ministério da Saúde, Comité Nacional de Bioética para a Saúde. MRC: The Gambia Government/MRC Joint Ethics Committee. NIMR-MMRC: Mbeya Medical Research and Ethics Committee. LMU: Ethikkommission bei der LMU München.

\section{Consent for publication}

Not applicable.

\section{Competing interests}

The authors declare that they have no competing interests.

\section{Publisher's Note}

Springer Nature remains neutral with regard to jurisdictional claims in published maps and institutional affiliations.

\section{Author details}

'Division of Infectious Diseases and Tropical Medicine, Medical Centre of the University of Munich (LMU), Munich, Germany. ${ }^{2}$ German Center for Infection Research (DZIF), Partner Site Munich, Munich, Germany. ${ }^{3}$ The Aurum Institute, Johannesburg, South Africa. ${ }^{4}$ Instituto Nacional de Saúde (INS), Ministry of Health, Maputo, Mozambique. ${ }^{5}$ Medical Research Council Unit The Gambia, Banjul, The Gambia. 'Department of Medicine, Imperial College London, London, UK. ${ }^{7}$ NIMR - Mbeya Medical Research Centre, Mbeya, Tanzania. ${ }^{8}$ Health Economics and Epidemiology Research Office, Department of Internal Medicine, School of Clinical Medicine, Faculty of Health Sciences, University of the Witwatersrand, Johannesburg, South Africa. ${ }^{9}$ Department of Public Health Sciences, Karolinska Institutet, Stockholm, Sweden. ${ }^{10}$ Research Center Borstel, Borstel, Germany. ${ }^{11}$ Clinical HIV Research Unit, Department of Internal Medicine, School of Clinical Medicine, Faculty of Health Sciences, University of the Witwatersrand, Johannesburg, South Africa. ${ }^{12} \mathrm{School}$ of

Public Health, University of Witwatersrand, Johannesburg, South Africa.

${ }^{13}$ Advancing Care and Treatment for TB/HIV, South African Medical Research Council, Parktown, Johannesburg, South Africa.

Received: 6 February 2018 Accepted: 28 December 2018

Published online: 07 January 2019

\section{References}

1. WHO. Global tuberculosis report 2017. Geneva: World Health Organization; 2017.

2. WHO. Global tuberculosis report 2012. Geneva: World Health Organization; 2012. 
3. Murray CJL, Vos T, Lozano R, Naghavi M, Flaxman AD, Michaud C, et al. Disability-adjusted life years (DALYs) for 291 diseases and injuries in 21 regions, 1990-2010: a systematic analysis for the global burden of disease study 2010. Lancet Elsevier. 2012;380:2197-223.

4. WHO. Global tuberculosis report 2013. Geneva: World Health Organization; 2013.

5. Ralph AP, Kenangalem E, Waramori G, Pontororing GJ, Sandjaja TE, et al. High morbidity during treatment and residual pulmonary disability in pulmonary tuberculosis: under-recognised phenomena. PLoS One. Public Libr Sci. 2013:8:e80302.

6. Akkara SA, Shah AD, Adalja M, Akkara AG, Rathi A, Shah DN. Pulmonary tuberculosis: the day after. Int J Tuberc Lung Dis. 2013;17:810-3.

7. Ehrlich Rl, Adams S, Baatjies R, Jeebhay MF. Chronic airflow obstruction and respiratory symptoms following tuberculosis: a review of south African studies [review article]. Int J Tuberc Lung Dis. 2011;15:886-91.

8. WHO. Global tuberculosis report 2015. Geneva: World Health Organization; 2015.

9. Lönnroth K, Roglic G, Harries AD. Improving tuberculosis prevention and care through addressing the global diabetes epidemic: from evidence to policy and practice. Lancet. Diabetes Endocrinol. 2014;2:730-9.

10. Jeon CY, Murray MB. Diabetes Mellitus Increases the Risk of Active Tuberculosis: A Systematic Review of 13 Observational Studies. Williams B, editor. PLoS Med. Public Libr Sci; 2008;5:e152.

11. Baker MA, Harries AD, Jeon CY, Hart JE, Kapur A, Lönnroth $K$, et al. The impact of diabetes on tuberculosis treatment outcomes: A systematic review. BMC Med. BioMed Central Ltd; 2011;9:81.

12. Creswell J, Raviglione M, Ottmani S, Migliori GB, Uplekar M, Blanc L, et al. Tuberculosis and noncommunicable diseases: neglected links and missed opportunities. Eur Respir J. 2011;37:1269-82.

13. Rehm J, Samokhvalov A V, Neuman MG, Room R, Parry C, Lönnroth K, et al. The association between alcohol use, alcohol use disorders and tuberculosis (TB). A systematic review. BMC Public Health. BioMed Central Ltd; 2009;9:450.

14. Lönnroth K, Williams BG, Cegielski P, Dye C. A consistent log-linear relationship between tuberculosis incidence and body mass index. Int J Epidemiol. 2010;39:149-55.

15. Dallenga T, Repnik U, Corleis B, Eich J, Reimer R, Griffiths GW, et al. M. tuberculosis -Induced Necrosis of Infected Neutrophils Promotes Bacterial Growth Following Phagocytosis by Macrophages. Cell Host Microbe. 2017 22:519-30 e3.

16. Eum SY, Kong JH, Hong MS, Lee YJ, Kim JH, Hwang SH, et al. Neutrophils are the predominant infected phagocytic cells in the airways of patients with active pulmonary TB. Chest. 2010;137:122-8.

17. Berry MPR, Graham CM, McNab FW, Xu Z, Bloch SAA, Oni T, et al. An interferon-inducible neutrophil-driven blood transcriptional signature in human tuberculosis. Nature. 2010;466:973-7.

18. Portevin D, Moukambi F, Clowes P, Bauer A, Chachage M, Ntinginya NE, et al. Articles assessment of the novel T-cell activation marker - tuberculosis assay for diagnosis of active tuberculosis in children : a prospective proof-of-concept study. Lancet. 2014;3099:1-8.

19. Ota MOC, Mendy JF, Donkor S, Togun T, Daramy M, Gomez MP, et al. Rapid diagnosis of tuberculosis using ex vivo host biomarkers in sputum. Eur Respir J. 2014;44:254-7.

20. Comas I, Gagneux S. A role for systems epidemiology in tuberculosis research. Trends Microbiol. 2011;19:492-500.

21. Zhang Y, Yew WW, Barer MR. Targeting persisters for tuberculosis control. Antimicrob Agents Chemother. 2012;56:2223-30.

22. Mitchison DA, Nunn AJ. Influence of initial drug resistance on the response to short-course chemotherapy of pulmonary tuberculosis. Am Rev Respir Dis. 1986;133:423-30.

23. Wallis RS, Wang C, Meyer D, Thomas N. Month 2 Culture Status and Treatment Duration as Predictors of Tuberculosis Relapse Risk in a MetaRegression Model. Via LE, editor. PLoS One. Public Libr Sci; 2013;8:e71116.

24. Barter DM, Agboola SO, Murray MB, Bärnighausen T. Tuberculosis and poverty: the contribution of patient costs in sub-Saharan Africa - a systematic review. BMC Public Health. 2012;12:980.

25. Tanimura T, Jaramillo E, Weil D, Raviglione M, Lönnroth K. Financial burden for tuberculosis patients in low- and middle-income countries: a systematic review. Eur Respir J. 2014;43:1763-75.

26. Lönnroth K, Glaziou P, Weil D, Floyd K, Uplekar M, Raviglione M. Beyond UHC: monitoring health and social protection coverage in the context of tuberculosis care and prevention. PLoS Med. Public Libr Sci. 2014;11:e1001693.
27. Tuberculosis patient cost surveys: a hand book. Geneva: World Health Organization; 2017. Licence: CC BY-NC-SA 3.0 IGO.

28. Hnizdo E, Singh T, Churchyard G. Chronic pulmonary function impairment caused by initial and recurrent pulmonary tuberculosis following treatment. Thorax. 2000;55:32-8.
Ready to submit your research? Choose BMC and benefit from:

- fast, convenient online submission

- thorough peer review by experienced researchers in your field

- rapid publication on acceptance

- support for research data, including large and complex data types

- gold Open Access which fosters wider collaboration and increased citations

- maximum visibility for your research: over $100 \mathrm{M}$ website views per year

At BMC, research is always in progress.

Learn more biomedcentral.com/submissions 